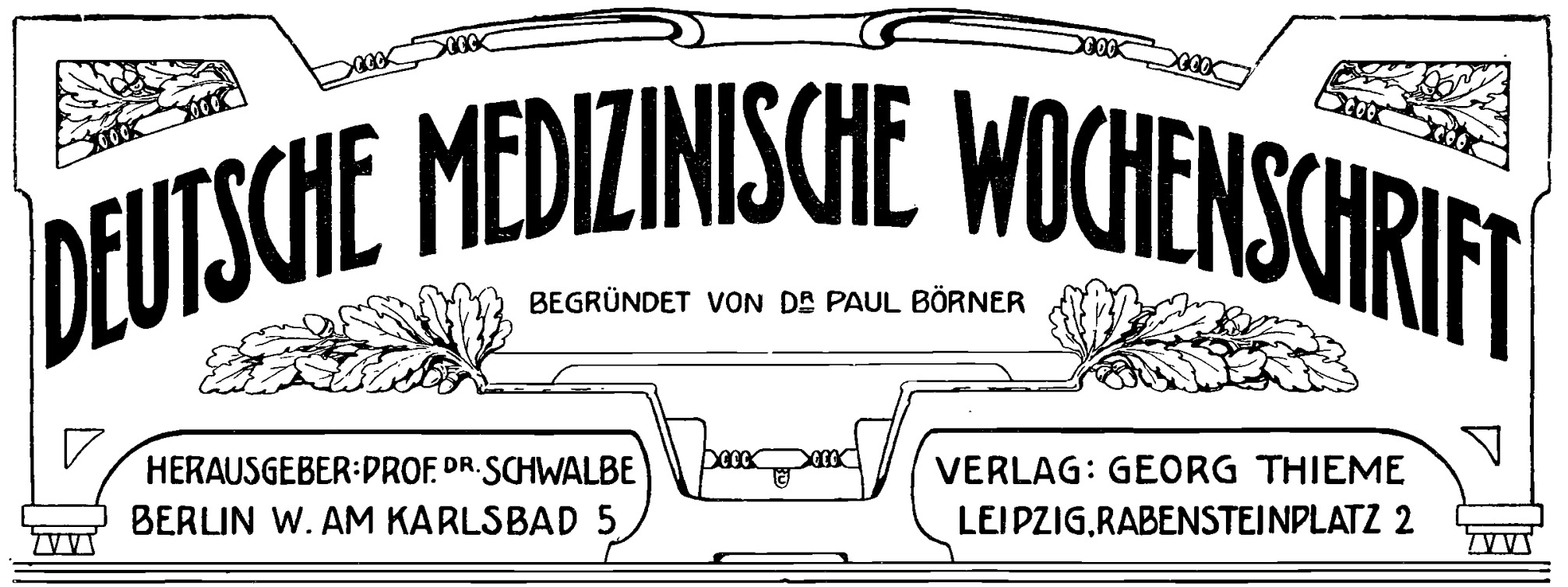

№ᅳ 17

BERLIN, DEN 29. APRIL 1909

35. JHHRGRNG.

\section{Zur Physiologie und Pathologie des Dickdarms.}

Von Prof. Dr. Th. Rosenheim.

Die bessere Einsicht, die wir in den letzten Jahren in die Anatomie und Physiologie des Magendarmkanals gewonnen haben, hat es uns ermöglicht, auch pathologische Vorgänge in den verschiedenen Abschnitten richtiger zu verstehen. Bleiben auch die einzelnen Teile des langgedehnten Organs eine Einheit, weil sie eine gemeinschaftliche Aufgabe haben, den gleichen Einflüssen vom Nervensystem, von anderen Organen, wie von außen her unterliegen, im gesunden und kranken $\mathrm{Zu}$ stande dauernd voneinander abhängig sind und aufeinander einwirken, so zeigt doch anderseits jeder Abschnitt ein hohes MaB von Eigenart und Selbständigkeit. Das erkennen wir auch am Kolon, dessen Funktionen man bis vor kurzem noch ziemlich schematisch einseitig und einförmig auffaßte. Und doch ist die Arbeit des Coecums in der Norm weit verschieden von der des $\mathrm{S}$ romanum, die ihrerseits selbst von der des Colon tranversum noch erheblich abweicht. Demgemäß reagieren die einzelnen Partien bei Reizungen mit verschiedenen Symptomen; sie zeigen sich für diese oder jene Störung verschieden empfänglich.

Ich $\left.{ }^{1}\right)$ habe schon im Jahre 1904 auf die Sonderstellung hingewiesen, die dem $S$ romanum im Vergleich zu den höheren Kolonteilen zukommt: ich betonte die verhältnismäßig reichliche und andauernde Füllung dieses Abschnitts mit festen Ballen schon in der Norm infolge einer erheblichen Verlangsamung des Kotlaufs im Gegensatze zu den höheren Partien mit Ausnahme des Coecums, bei dem der regelmäßigere Befund von Kotbrei durch die Besonderheit seiner Funktion begreiflich ist; es ist eben ein Reservoir, in das fast kontinuierlich ein rasches Zuströmen von dünnen Massen durch die Bauhinsche Klappe statthat und in dem sie sich stauen. Ich machte auch schon damals auf den verhältnismäßig geringen Gasgehalt der Sigmaschlinge aufmerksam; echte, klinisch erweisbare Tympanie, die sich in der oberen Dickdarmteilen so häufig beobachten läßt, ist hier etwas Ungewöhnliches; sie findet sich eigentlich nur beim Volvulus, gelegentlich bei Strikturen und bei entzündlichen und geschwürigen Prozessen, wenn sie mit DFarrhöen einhergehen; ich sah sie in den letzten Jahren zweimal bei hochgradig nervösen, chronisch obstipiertén Frauen vorübergehend mit gleichzeitiger extremer Ausweitung der Ainpulla recti: die leere Schlinge markierte sich bei der Palpation und Perkussion, als wäre eine Aufblähung mit Luft künstlich vom Mastdarm aus vorgenommen. Sicher bestand hier kein anatomisches Hindernis, keine Striktur, sondern eine idiopathische Darmmuskelparese hohen Grades, sodab schon gelinder Gasdruck, wie er sonst nicht in diesem muskelkräftigsten Teil des Kolons zur Erzeugung einer Tympanie ausreicht, das Organ überdehnte. Die endoskopische Untersuchung, in dem einen

1) Zeitschrift für klinische Medizin, Bd. 54 . der beiden Fälle ausgeführt, bestätigte die Diagnose einer diffusen atonischen Dilatation des Mastdarms und der Flexura sigmoidea.

Es ist ein besonderes Verdienst von Roith, ${ }^{1}$ ) die Füllungsverhältnisse des Dickdarms an Leichen und bei der Autopsie in vivo genau studiert zu haben. Seine Ergebnisse decken sich in bezug auf Coecum und Sigmaschlinge im wesentlichen mit den meinen, die durch Palpation am Lebenden, ein immerhin unsichereres Verfahren, gewonnen wurden. Roith war in der Lage, über das hinaus, was ich feststellen konnte, einige Tatsachen zu eruieren, z. B. die Sonderstellung, die das Colon descendens einnimmt, da es meistens leer gefunden wird, an Kot- und Gasgehalt von der Flexura sigmoidea stets weit übertroffen wird, die den proximalen Dickdarmabschnitten (Coecum + ascendens + transversum) darin ähnlicher ist, aber im ganzen den letzteren, absolut und relativ berechnet, nachsteht. Es ergibt sich also eine auffallende Differenz in den Füllungs- und zugleich in den Druckverhältnissen des Kolons vor und unmittelbar hinter der Flexura Coli sinistra, und mit Recht wird von Roith ein wesentlicher Einfluf auf das Zustandekommen dieses Phänomens dem Mechanismus zugeschrieben, der gegeben ist einmal in der straffen Fixation des Kolons an der Biegungsstelle links hinten oben und dann in der spitzwinkligen Knickung des Darmes, die bei größerer Spannung sich ungünstig verstärkt, ja zu klappenförmigem Verschluß durch das Vorspringen einer Falte an der konkaven Wand führen kann. Aus den Füllungsverhältnissen des Darmes lassen sich aber auf die Raschheit der Fortbewegung der Massen in seinen verschiedenen Partien nur sehr bedingte Schlüsse ziehen. Zwar wird man auf Grund obiger Beobachtungen annehmen dürfen, da@ die Beförderung durch das Colon descendens relativ rasch und jedenfalls schneller als in höheren Abschnitten erfolgt, aber ob die geringere Ansammlung von Darminhalt im S romanum als im Coecum und Colon ascendens die Auffassung rechtfertigt, daß hier die Contenta träger als dort vorwärtsgeschoben werden, ist mehr als zweifelhaft. Zwar nimmt die Muskulatur vom Coecum zum Rectum an Stärke $\mathrm{zu}$, während das Lumen gleichzeitig enger wird, aber es ist doch zu berücksichtigen, daß das Wesentliche in bezug auf Resorption und Assimilation an der Flexura coli sinistra bereits geleistet ist und daß unterhalb derselben die geformten soliden Kotstücke eine erheblich größere Muskelarbeit zu ihrer Fortbewegung erheischen als die breiig weichen Contenta im rechtseitigen und queren Teil des Dickdarms. Man könnte meinen, daß die Anwendung der Röntgenstrahlen am mit Bismut gefüllten Darm uns eine zuverlässige Kontrolle der Vorwärtsbewegung des Inhalts ermöglicht, und die Untersuchungen von Rieder, $\left.{ }^{2}\right) \mathrm{Hertz}^{3}$ ) $\mathrm{u}$. a. haben uns auch manches Beachtenswerte gezeigt, aber wir müssen be-

1) Anatomische Hefte, Bd. 20; Medizinische Klinik 1906, No. 1 u. 2; Mitteilungen aus den Grenzgebieten der Medizin und Chirurgie, Bd. 19. - 2) Fortschritte auf dem aus den Grenzgebieten der Medizin und Chirurgie, Bd. 19. - 2) Fortschritte auf dem
Gebiete der Röntgenbehandlung, Bd. 8 u. 10. - 3) Arch. des maladies de l'appareil diGestif, 1908 . 
denken, daß der Bismut auberordentlich lange im Kolon verweilt (3-4 Tage), der Schleimhaut zähe anhaftend, und daß er adstringierend und kotlaufhemmend wirkt, sodab alle Resultate nur mit größter Vorsicht zu verwerten sind. So werden wir wohl sicher über die Schnelligkeit, mit der bismuthaltiger Chymus die einzelnen Etappen erreicht, belehrt, aber nicht zuverlässig über die Kotfüllungsverhältnisse der verschiedenen Teile und ganz unvollkommen über die Aufenthaltsdauer der Faeces in dieser und jener Partie, denn der Bismut zeigt unter Umständen einen Schatten an auch dort, wo wenig oder gar keine Scybala mehr vorhanden sind, da er länger haften kann als die Reste der Durchschnittsnahrung. Immerhin verdient die Bemerkung Rieders Beachtung, daß unabhängig von der Qualität der Nahrung bismuthaltige Massen sehr lange im Coecum bleiben und daß Stauung von Inhalt außer im Coecum und Colon ascendens sehr häufig im S romanum und auch im Rectum konstatiert wird; desgleichen erscheint die Beobachtung von Hertz belangreich, daß bei der normalen Defäkation eine völlige Entleerung des Darms bis hinauf zur Flexura coli sinistra statthat, während gleichzeitig auch in den rechtseitig gelegenen Abschnitten eine energischere Vorwärtsbewegung sich geltend macht.

Alle diese Feststellungen decken sich grobenteils mit Ergebnissen meiner eigenen Untersuchungen, über die ich schon früher berichtete und die ich jetzt vervollständigen kann. Ich habe systematisch im Laufe der letzten Jahre eine Anzahl gesunder Personen palpatorisch mehrmals am Tage in bezug auf die Füllungsverhältnisse ihres Darmkanals untersucht. Es waren meist jugendliche Individuen, deren Magerkeit eine eingehende Prüfung erleichterte und die gewohnheitsmäßig auf den Glockenschlag nach dem ersten Frühstück ihren Stuhlgang einmal am Tage absetzten. Die Untersuchungen wurden $\mathrm{zu}$ verschiedenen Stunden, auch des Morgens nüchtern, an mehreren aufeinanderfolgenden Tagen vorgenommen, bei manchen Personen erstreckte sich die Beobachtung über Jahr und Tag. Die Palpation des Coecum resp. Colon ascendens, der Flexura sigmoidea resp. des Colon pelvicum (Untersuchung in Steinschnittlage) und die des Rectum ergab wohl stets zuverlässige Resultate; unsicherer waren dieselben am Colon transversum, das öfter entweder nur teilweise, gelegentlich auch mal überhaupt nicht getastet werden konnte. Ohne auf Einzelheiten einzugehen, möchte ich das Ergebnis dahin zusammenfassen:

1. Wenn die letzte Mahlzeit abends zwischen 8-10 Uhr genommen war, so wurde das Coecum und Colon ascendens morgens nüchtern meistens leer gefunden; es war gewöhnlich leicht kontrahiert, seltener mittelstark gebläht. Zu dieser Zeit war die Flexura sigmoidea stets mit Kotmassen prall gefüllt. Nur in einem Falle war sie fast immer leer; hier fanden sich die Massen bereits im Rectum. Auch dort, wo die Flexur, vom Abdomen her palpiert, sich gefüllt erwies, konnten bereits Massen im Colon pelvicum, im oberen Rectumdrittel, manchmal auch in der Ampulle nachgewiesen werden.

2. Wenn der Defäkationsakt erfolgt war, so wurde nicht blob das Rectum und die Flexura sigmoidea, sondern auch das Colon descendens leergefunden; die letztgenannten höheren Abschnitte zeigten sich dagegen bei einem Gesunden, der gewohnheitsmäßig zweimal in 24 Stunden zu Stuhl ging, mehrfach unvollkommen entleert. In den Füllungsverhältnissen des Querkolons, so oft dasselbe palpierbar war, konnte niemals eine Veränderung durch den Defäkationsakt als solchen deutlich nachgewiesen werden.

3. Im Laufe des Tages, etwa mittags, ungefähr fünf Stunden nach dem ersten Frühstück, konnte im Coecum weichbreiiger Inhalt festgestellt werden. Die Coecalfüllung blieb bis zur Nacht bestehen und dürfte also erst während derselben allmählich verschwunden sein. Der Gasgehalt des Blinddarms wechselte bei den verschiedenen Individuen in weiten Grenzen; vorübergehend war er auch hier in der Norm recht beträchtlich. Sehr viel geringer war eine Auftreibung am Colon ascendens, und noch weniger ausgesprochen und unregelmäßiger konnte sie am Colon transversum beobachtet werden. Die Flexura sigmoidea war öfters schon nach. mittags vor 6 Uhr deutlich mit Kotstücken gefüllt, in den späteren Abendstunden war sie es ausnahmslos; der Gasgehalt war zu jeder Zeit gering. Das Rectum war abends leer, nur hin und wieder fanden sich kleine Bröckel.

Ich kann also aussagen, dab normalerweise das Coecum nüchtern zwar nicht immer, wie Herz meint, aber doch gemeinhin leer ist, daß es etwa 5 Stunden nach der ersten Mahlzeit eine Füllung zu zeigen pflegt und daß bei einer ausreichenden Defäkation die Entleerung sich nicht blob auf das Rectum, sondern auf die ganzen Abschnitte unterhalb der Flexura coli sinistra erstreckt. Ich mub von neuem, worauf ich schon vor Jahren hinwies, die auffallende Tatsache hervorheben, dab die Beförderung im Bereiche der Flexura sigmoidea eine sehr langsame ist, daß hier ein direkter Aufenthalt der Fäkalmasse statthat und dab dieses Organ länger als 12 Stunden des Tages Kot beherbergt und dessen Einwirkungen ertragen mub. Würde die Beförderung des Darminhaltes in der Flexura gleichmäfig schnell vor sich gehen, so würde ein kontinuierliches Zuströmen in das Rectum statthaben, was wohl den Zwang zu häufigerer Stuhlentleerung auch beim Gesunden tagüber zur Folge haben würde. So aber, wie sich die Dinge gestalten, hat man den Eindruck, daß das $S$ romanum in gewissen Grenzen als Reservoir dient, eine Auffassung, die ich auch in dem soeben erschienenen Buch von 0. Cohnheim ${ }^{1}$ ) ausgesprochen finde. Die Kräfte, welche es ermöglichen, daß hier physiologisch eine gewisse Hemmung zustande kommt, sind einmal gegeben in dem eigenartigen anatomischen Bau des Colon pelvicum, dessen Fixation innerhalb des kleinen Beckens am sich verkürzenden Mesenterium eine solche zu sein pflegt, daß es gefaltet zusammenliegt und daß es im Winkel zum Rectum steht und etwas schräg in dasselbe einmündet. So erklären sich auch die Schwierigkeiten, die wir an der Plica rectoromana beim Endoskopieren gemeinhin finden und die wir überhaupt erst durch bestimmte Lagerungen des Patienten und Drehungen des Tubus zu überwinden imstande sind. So erklären sich aber auch die eigentümlichen Bilder, die wir am Eingang in die Flexur, wenn sie noch Kotmassen enthält, häufig zu sehen bekommen. Wir beobachten straff gespannte, fest vorgelagerte Falten oder krampfhaften, rosettenartigen Verschluf und haben den Verdacht des Vorliandenseins pathologischer Zustände, während wir in Wirklichkeit nur ein deutliches Bild davon bekommen, wie der normale Darm die Scybala an dieser Stelle festhält. Anscheinend wirken neben den begünstigenden anatomischen Verhältnissen auch ganz bestimmte Innervationsvorgänge ordnungsgemäb hemmend. Zunächst stellt sich hier wohl der peristaltische Impuls nur selten und jedenfalls seltener als in den oberen Teilen ein, zweitens scheint die Ringmuskulatur hier länger und kräftiger innerviert zu werden als die Längsmuskulatur, woraus eine gleichmäßigere anhaltende tonische Kontraktion des Organabschnittes resultiert, und schließlich dürfte die Vermutung von Roith ${ }^{2}$ ) wohl begründet sein, daß auch hier, wie dies ja für das obere Dickdarmdrittel zweifellos nach den Untersuchungen von Jacobi, Cannon, Elliott und Barclay-Smith, Magnus, Roith feststeht, eine Antiperistaltik schon in der Norm den Kotlauf beeinflubt.

Aus den bisherigen Untersuchungen über den Kotlauf im gesunden Dickdarm ergibt sich nun, daß die Füllungsverhältnisse des Organs zu verschiedenen Tageszeiten, vor und nach der Defäkation nicht allemal schematis ch dieselben sind, sondern in gewissen Grenzen schwanken können, wie dies auch garnicht anders zu erwarten ist, da überall innerhalb der physiologischen Breite mancherlei Differentes zu Recht besteht. Der Befund von Hertz beispielsweise, daß das Coecum nüchtern leer ist, wenn es auch für die Mehrzahl der Fälle zutrifft, läßt sich nicht verallgemeinern (s. oben); unübersehbare Hemmungen (Antiperistaltik) können auch unter normalen Verhältnissen eine gewisse Ungleichmäßigkeit der Funktion der einzelnen Dickdarmteile bedingen. Ebensowenig findet sich Füllung der Flexura sigmoidea vor dem Defäkationsakt unter allen Umständen, auch das Rectum kann in dieser Phase als Reservoir dienen, und die Auffassung, daß

1) Die Physiologie der Verdauung und Ernährung 1908, S. 39

2) Mitteilungen aus den Grenzgebieten, Bd, 19. 
die Füllung der Ampulle gesetzmäßig den Stuhlakt einleitet, ist nicht allemal zutreffend. Und vielleicht wird sich auch mal bei einem Gesunden nachweisen lassen, daß nach einer anscheinend völlig befriedigenden Stuhlabsetzung doch noch geringe Reste von Kot unterhalb der Flexura coli sinistra zurückbleiben: individuelle Verhältnisse, Gewöhnung und Anpassung bedingen funktionelle Differenzen und bewirken wechselnde Befunde. Aber mit der nötigen Kritik werden wir trotzdem gewisse erheblich abweichende Untersuchungsergebnisse als nicht mehr normale, als pathologische ansprechen dürfen. Von diesem Gesichtspunkt ausgehend, habe ich mich seit Jahren bemüht, palpatorisch die Füllungsverhältnisse des Darms bei meinen Patienten mit chronischer Obstipation eingehend zu prüfen.

Ich suchte festzustellen, ob sich nicht aus der groben Zahl von Fällen, die unter der Sammeldiagnose „habituelle Obstipation“ gemeinhin geführt werden, verschiedene Gruppen auslesen lassen, denen eine gewisse Sonderstellung und Selbständigkeit zuerkannt werden darf,indem die Palpation uns ganz bestimmte differentielle Merkmale lehrt. Ich achtete dabei zunächst auf Unterschiede des gasigen und festen Inhalts der verschiedenen Dickdarmteile, des weiteren auf die Energie der Kontraktion des Darmmuskels. So nützlich die hierbei gemachten Beobachtungen manchmal waren, einen entschiedenen Fortschritt in der Erkenntnis des Charakters und des Zustandekommens der Obstipation im gegebenen Falle, eine Förderung in der Behandlung der einschlägigen Zustände ergab sich doch nur selten. Ich überzeugte mich bald, daß die so beliebte schematische Trennung der Obstipationen in atonische und spastische undurchführbar war. $\mathrm{Da} \beta$ atonische und spastische Zustände, deren Resultat gleicherweise Verstopfung ist, im Kolon vorkommen, leugne ich natürlich nicht. Ich gehe auch nicht so weit, das Vorkommen einer rein spastischen Obstipation, wie dies jüngst von $\mathrm{Boas}^{1}$ ) geschehen ist, zu verneinen - sie kommt vor, aber sie ist sicher selten; nur haben wir es gemeinhin bei den habituell Verstopften mit komplexen Störungen zu tun, sodaß im Einzelfall Atonie und Spasmus nebeneinander bestehen und aufeinander folgen können. Bedenken wir doch, dab der Bewegungsmodus am Dickdarm ein überaus mannigfaltiger ist, wir kennen an ihm Pendel, richtiger Mischbewegungen, dann Peristaltik und Antiperistaltik, endlich sogar tonische Kontraktionen der Muskulatur schon in der Norm. Berücksichtigen wir, wie unübersehbar kompliziert die Innervationsvorgänge sind, von wie vielen im Darm und außerhalb desselben gelegenen Faktoren sie beeinflubt werden, so ist es ohne weiteres klar, daß wir das Wesen einer Kotstauung nicht aus dem Verhalten der Muskulatur, wie es sich bei der Palpation darstellt, erschließen können.

Es kommt hinzu, daß man bisher viel zu leicht geneigt war, ganz physiologische Zustände der Muskulatur, die das Bild einer Atonie oder eines Spasmus darboten, als pathologisch anzusprechen. Das gilt z. B. von der Diagnose der sogenannten spastischen $\mathrm{Ob}$ stipation auf Grund des Befundes einer fest um ihren Inhalt kontrahierten Sigmaschlinge. Die wenig verbreitete Kenntnis der Funktion dieses Organabschnitts unter normalen Verhältnissen und der Art ihrer Arbeitsleistung macht diesen Irrtum verständlich. Man erinnere sich dessen, was oben von der Bedeutung des S romanum als Reservoir gesagt wurde, man gedenke des Vorkommens tonischer Kontraktion in seinem Verlauf, des Auftretens von Spasmen zur Zurückhaltung der Massen in seinem untersten Stück, worauf ich oben noch spezieller hinwies, so ergibt sich, wie vorsichtig wir mit der Diagnose einer durch abnorme Krampfneigung der Muskulatur bedingten Obstipation sein müssen. Und ebenso häufig wie der Befund des Spasmus im untersten Kolonabschnitt in der Norm ist, ebenso häufig begegnen wir einem atonischen Zustande der oberen Dickdarmhälfte beim Gesunden und bei bester Leistungsfähigkeit. Ballonartige Auftreibung des Coecums, erheblichere Gasspannung im Colon ascendens, seltener im Querdarm beobachten wir bei normaler Verdauung, ohne daß die geringsten subjektiven Erscheinungen auf das Phänomen hinweisen. Art der Lebensführung und Qualität

1) Medizinische Klinik 1908, No. 39. der Nahrung, individuelle Besonderheit in bezug auf Zersetzungsvorgänge im Darm, in bezug auf Elastizität des Darmschlauches, in bezug auf Energie der Innervation bewirken hier erhebliche individuelle Schwankungen in der physiologischen Breite.

Worauf ich also keinen erheblichen Wert für die Beurteilung und speziell auch für die Behandlung eines Falles von chronischer Obstipation legen möchte, das ist die Diagnose des jeweiligen, öfter wechselnden Zustandes der Muskulatur in diesem oder jenem Abschnitt des Darms. Dagegen halte ich für entscheidend wichtig eine genaue Kontrolle der Füllungsverhältnisse des 0rgans in seinen einzelnen Teilen. Die Frage ist: ist das, was wir unter dem Namen habituelle Verstopfung zu begreifen pflegen, ausnahmslos oder auch nur in der Mehrzahl der Fälle eine Erkrankung des ganzen Dickdarmschlauches? Sie muß bestimmt verneint werden. Schon seit langem wissen wir, dal andauernde chronische Obstipation ihren Grund haben kann in einer Störung der Mastdarmfunktion allein: durch das Kolon hindurch werden die Massen prompt bis ins Rectum hineinbefördert, und hier stocken sie, sei es infolge Verlustes des Stuhldrangsgefühls, sei es infolge einer Erschlaffung resp. Erweiterung der Ampulle, sei es infolge einer Schädigung der quergestreiften Muskulatur des Beckenbodens, sei es infolge von Entzündung und Fissurenbildung im Aftergebiet, sei es infolge einer Erkrankung des benachbarten Genitalapparates und noch aus anderen Gründen. Aber auch für das Kolon bin ich in der Lage den Nachweis zu führen, dab partielle lokalisierte Schädigungen seiner Funktion vorkommen, die Kotstockung in demselben begrenzten Abschnitt des Organs zur Folge haben, dal es beispielsweise 0 bstipationen gibt, die ausschlieb. lich in der Flexura sigmoidea oder im Coecum resp. Colon ascendens in die Erscheinung treten und die symptomatisch und therapeutisch sich durchaus verschieden verhalten. Aber auch die mittleren Abschnitte des Kolons, besonders die Flexuren, können für sich allein Sitz der Störung sein.

Die Diagnose dieser Zustände ermöglicht uns die Palpation, wiederholt mit der nötigen Sorgfalt im gegebenen Falle geübt. Wollen wir uns ein klares Bild vom Sitz der Hemmung verschaffen, so müssen wir den Patienten natürlich im Stadium der Verstopfung untersuchen: ich lasse den Kranken zwei bis drei Tage seine Kotverhaltung ertragen, bei Aufnahme einer milden, gemischten, in keiner Beziehung einseitigen Kost, wie sie ein Durchschnittsmensch gut aufnimmt und bei der er regelmäßig Stuhlgang zu haben pflegt. Die Wirkung gewohnheitsmäßig genommener Abführmittel und Einläufe muß abgeklungen sein, damit wir ein Urteil darüber zu gewinnen vermögen, was der Darm noch aus eigener Kraft zu leisten imstande ist. Den Protest der Patienten, daß sie einige Tage nicht verstopft bleiben könnten, daß sie unangenehme Folgen zu gewärtigen hätten, wird man gemeinhin unbeachtet lassen können. Sie mögen leichte Verstimmungen und Unbequemlichkeiten bei unserer Disposition hin und wieder zu ertragen haben; aber das können sie wohl im Interesse der Klarstellung ihres hartnäckigen Leidens in den Kauf nehmen, und eine solche läßt sich in vielen Fällen tatsächlich durch die auf der Höhe der Stauung erfolgende, exakt durchgeführte Palpation erzielen. Es ist selbstverständlich von großer Bedeutung und dringend wünschenswert, das Ergebnis durch Kontrolluntersuchungen zu sichern. Dal die Methode nicht selten, z. B. bei Adipositas unvollkommene Resultate ergibt, daß sie allemal mit Kritik anzuwenden ist, bedarf keiner besonderen Hervorhebung.

Es hat sich nun herausgestellt, daß eine beträchtliche Zahl habitueller Obstipationen ausschlieblich auf einer Störung der Funktion der Flexura sigmoidea beruht. Kaum je habe ich in diesen Fällen einen Zustand des Organs festzustellen vermocht, den man als atonischen bezeichnen könnte. Meist war das Organ fest um seinen Inhalt geschlossen; es bestand eine gleichmäßjge tonische Kontraktion. Neben dieser oder auch für sich allein, besonders im Gebiete des Colon pelvicum, war häufiger eine Zerbröckelung der retinierten Kotmassen $\mathrm{zu}$ konstatieren, sodab fest abgegrenzte 
Kugeln, um die der Muskel sich krampfhaft schloß, tastbar waren. Ich habe deshalb, was ich schon früher andeutete, den Eindruck, daß, was so gemeinhin unter der Devise spastische Obstipation geht, auf einer Kotlaufhemmung im Gebiete der Flexura sigmoidea schlechtweg beruht.

Weniger häufig sind Coecum und Colon ascendens, die hier nach meinen Beobachtungen zusammengehören und eine Einheit bilden, ausschließlicher Sitz einer deutlich palpatorisch erweisbaren Kotlaufhemmung. Dabei sind die Befunde wechselnde. In einem Teil der Fälle vermag man mehr oder weniger umfangreiche härtere Scybala zu tasten, um die sich das Organ verschieden straff kontrahiert. Gelegentlich bestehen auch hier echte, hartnäckig andauernde Spasmen.

Andere Male ist die Gasansammlung in diesem Darmstück so beträchtlich, daß dasselbe beutelartig prominiert und die festeren Kotmassen nur schwer oder auch garnicht durchzufühlen sind. Der Befund von harten Fäkalstücken im Blinddarm ist, wie ich nicht erst zu sagen brauche, unter allen Umständen pathologisch. Schließlich kann das Palpationsergebnis zu verschiedenen Tageszeiten wechseln: man findet des Morgens den obersten Dickdarmabschnitt schlaff, gashaltig und in den Abendstunden seine Wand fest um die soliden Kotpartikel zusammengezogen

Noch seltener sind partielle Kotlaufhemmungen an den Flexuren; sie sind auch schwerer zu diagnostizieren. Am charakteristischsten ist da noch der Befund an der rechten Seite; zwischen rechtem unteren Nierenpol und Nabel fühlt man eine um ihren Inhalt fest kontrahierte, voluminöse Schlinge. Die Flexura coli sinistra ist der Palpation nicht zugänglich, wohl aber die oralwärts gelegenen Teile des Colon transversum, die dann ebenfalls die Kotstauung erkennen lassen. Gasige Auftreibung dieser Partie ist seltener, häufiger findet sich die Coecalpartie sekundär gebläht und druckempfindlich.

Entscheidend für die Beurteilung im gegebenen Falle ist nun nicht blob der Nachweis der Kotansammlung an dieser oder jener Stelle, sondern auch die verhältnismäßige Leere der tieferen Abschnitte. Da nun aber besonders bei nervösen Individuen die funktionelle Leistungsfähigkeit in verschiedenen Abschnitten des Kolons aus zum Teil unkontrollierbaren inneren Ursachen wechseln kann, so hat erst die Konstanz des Befundes bei mehrmaliger Untersuchung zwingende Beweiskraft. Es ist auch ohme weiteres klar, daß Kotlaufhemmungen zu gleicher Zeit an verschiedenen Stellen zustande kommen können, wodurch das Bild verwischt wird; meist wird sich dann die Störung in der obersten Partie am schärfsten und charakteristischsten markieren. So findet sich $z$. B: eine Ansammlung von Fäkalmassen auf der rechten Seite und zugleich eine solche in der Sigmaschlinge oder im Rectum, während die mittleren Kolonpartien verhältnismäBig leer sind. Die Stagnation im untersten Dickdarmteil kann auch ein sekundärer Vorgang sein, denn hier und besonders in der Flexura sigmoidea machen sich hemmende Impulse reflektorisch, in Abhängigkeit von allen möglichen Störungen und Krankheitsherden in der Bauchhöhle auBerordentlich oft und schnell geltend. So begleitet Obstipation im Gebiete der Sigmaschlinge chronische Gastritis, Cholecystitis, Appendicitis garnicht selten, und so kann sie auch mal als Folge einer Kotstauung im oberen Dickdarmdrittel auftreten. Umgekehrt ist es nicht ausgeschlossen, daß die Hemmung im untersten Kolonteil ungünstig auf den Ablauf der Funktion im höheren zurückwirkt, ebenso wie beispielsweise die Pylorusarbeit vom Dünndarm her beeinflußt wird. Die Beziehungen und Zusammenhänge der Innervation des Magendarmschlauches sind eben äußerst mannigfaltige. Nach meinen Erfahrungen dürfte indes die letzterwähnte Kombination keine erhebliche praktische Bedeutung haben. Natürlich darf man sich durch das Phänomen der einfachen Rückstauung nicht täuschen lassen; diese kann z. B. bei primärer Kotlaufhemmung im $\mathrm{S}$ romanum auch noch das Colon transversum, ja sogar das Coecum, in den frühen Morgenstunden untersucht, gefüllt erscheinen lassen; aber die Hauptmasse der Scybala muß sich immer links finden, sonst ist die Annahme berechtigter, daß primär eine diffuse, so ziemlich das ganze Kolon gleichzeitig betreffende Schädigung vorliegt.

Die Verwertung der hier besprochenen Gesichtspunkte für die bessere Beurteilung der Fälle hartnäckiger Obstipation, insbesondere auch für therapeutische Zwecke befürworte ich in meinen Kursen seit Jahren. In der Praxis ist kaum je diese Auffassung zur Geltung gekommen; offenbar stand man immer zu sehr unter dem Einflub der Vorstellung, das ganze Kolon sei funktionell als Einheit zu betrachten: Physiologie und Pathologie haben das in den letzten Jahren als unhaltbar erwiesen, und es ist Zeit, daß die Klinik dem Rechnung trägt. Das Vorkommen partieller Entzündungen einzelner Kolonabschnitte ist heute eine gesicherte Tatsache: wir kennen echte akute und chronische Sigmoiditiden, wir verdanken $\mathrm{Payr}^{1}$ ) $\mathrm{u}$. a. ein besseres Verständnis für die umschriebene Entzündung in der Flexura coli sinistra, und die Coecalkatarrhe mit und ohne Appendicitis sind uns einigermaßen geläufig geworden. So wird man sich auch mit dem Gedanken des regelmä日igen Auftretens partieller funktioneller Dickdarmhemmungen befreunden, ein Gedanke, dem in der Literatur eigentlich bisher nur v. Federn, ${ }^{2}$ ) allerdings in zu einseitiger Form Beachtung zu schaffen suchte. Die große Rolle, die dieser Autor der partiellen Darmatonie in der Pathologie zuweist, kann ich ebensowenig gelten lassen, als ich die Art seiner Diagnosenstellung (z. B. durch leise Fingerperkussion) als zuverlässig anzuerkennen vermag. Immerhin liegt etwas Richtiges in seinen Beobachtungen. In jüngster Zeit hat A. F. Hertz ${ }^{3}$ ) bei mehreren hartnäckig Obstipierten das Verhalten des Darms mit Hilfe der Röntgenstrahlen studiert, auch er findet Fälle mit lokalisierter Störung neben solchen, bei denen er eine gleichmäßige Verlangsamung des Kotlaufs im ganzen Dickdarm diagnostiziert; die Differenzierung dieser verschiedenen Obstipationsformen erscheint auch ihm speziell vom therapeutischen Standpunkt wichtig. Es ist aber ohne weiteres klar, daß, abgesehen von andern Einwänden, die Umständlichkeit seines Verfahrens es für die Kontrolle der alltäglichen Fälle ungeeignet macht; demgegenüber läßt meine Methode an Einfachheit nichts zu wünschen übrig und ist, wie ich denke, mindestens ebenso zuverlässig.

Was wir auf dem angegebenen Wege in einer Anzahl von Fällen zu diagnostizieren imstande sind, ist das Bestehen einer lokalisierten Dickdarmstörung als Grundlage einer hartnäckigen habituellen Obstipation: Nicht die Ursache des Uebels stellen wir auf diese Weise fest, sondern seinen speziellen Sitz. Die Gründe, warum Kotlaufhemmung in diesem oder jenem Teil in die Erscheinung tritt, sind überaus mannigfaltige. Alles, was chronische Obstipation schlechtweg herbeizuführen vermag, kann auch gelegentlich partielle Darmstörung in irgend einem Abschnitt des Organs hervorrufen, aber die letztere Form der Stuhlverhaltung kommt nach meinen Erfahrungen doch unter bestimmten Umständen besonders gern zustande. Wo Obstipation als deuteropathischer Zustand, als Begleit- und Folgeerscheinung aller Arten von Störungen und Affektionen im Gebiete der Bauchhöhle besteht, da tritt sie häufig lokalisiert in umschriebenem Kolonbezirk auf: neben Magenaffektionen, Cholecystitis, chronischer Appendicitis, Genitalerkrankungen finden wir sie; des weiteren durchaus nicht selten, wo sie das vielleicht hervorstechendste, quälendste Symptom einer allgemeinen Neurose darstellt. Ferner prädisponieren gewisse Lageanomalien der Bauchorgane zu bestimmten lokalisierten Kotstauungen. Aber auch die habituelle Obstipation sensu strictiori nach Nothnagel, die auf einer abnormen nervösen Einstellung der Kolon- und Rectumperistaltik allein beruhen soll, kann sich als ganz umschriebene Funktionsstörung manifestieren. Wir werden also immer verschiedenen Ursachen nachgehen und Rechnung tragen muissen, wie es auch sonst bisher bei der Beurteilung eines Falles chronischer Obstipation geboten war. $\mathrm{DaB}$ wir außerdem bei der Diagnose der partiellen Dickdarmstörungen das Vorhandensein hyperästhetischer Zonen, die Art der subjektiven Erscheinungen, das Verhalten

1) Archiv für klinische Chirurgie, Bd. 77. - 2) Wiener Klinik, Wien 1891 und Wiener klinische Wochenschrift 1904, No. 28, S. 806 . - 3) Proceedings of the Royal Soc. of Medic., Februar 1908 und Arch. of the Roentgen Ray and allied Phenomena, June 1908 
des Stuhlgangs, den ganzen Allgemeinzustand zu berücksichtigen haben, daß alle Symptome nur mit größter Vorsicht verwendet werden dürfen, bedarf wohl keiner besonderen Hervorhebung. Wie bei unserm Verfahren, wenn man mit der nötigen Kritik vorgeht, sich für viele Fälle recht nützliche therapeutische Gesichtspunkte ergeben, das will ich später genauer darlegen.

Zusammenfassung. Die einzelnen Abschnitte des Kolons haben eine erhebliche Eigenart und Selbständigkeit unter normalen und pathologischen Verhältnissen. Am Kolon kommen lokalisierte Schädigungen seiner Funktion vor, die Kotstockung in demselben begrenzten Abschnitt zur Folge haben; es gibt beispielsweise obstipationen, die ausschlieblich in der Flexura sigmoidea oder solche, die nur im Coecum resp. Colon ascendens in die Erscheinung treten. Die Diagnose dieser Zustände ermöglicht uns meist die Palpation des Dickdarms im Stadium der Verstopfung. Wir orientieren uns so über die Füllungsverhältnisse des Darms und erkennen was er bei habitueller Obstipation noch aus eigener Kraft zu leisten imstande ist, ob eine partielle funktionelle Dickdarmhemmung überhaupt vorliegt und wo ihr Sitz ist. Hieraus ergeben sich für viele Fälle wertvolle therapeutische Gesichtspunkte. 